\title{
Quel régime politique pour les états Africains : le cas du Togo
}

\author{
Elisée Tikonimbé Koupokpa
}

Faculté de Droit, Université de Lomé, Togo

\begin{abstract}
As it is the case for most of the African countries, Togo is looking for a constitutional system that fits its political, social and cultural realities. The I9gos were considered as a period of democratic revival, and from those years onwards the constitutional organization was directed towards a parliamentary type of regime which was rightfully or wrongfully seen as an instrument whereby the head of state's powers were limited. However, the unfortunate result of this kinds of organization of the political powers leads to another constitutional system which is more likely to be presidential.
\end{abstract}

Key words: political regime, parliamentary Regime, presidential regime, head of state, president of the republic, prime minister, counterbalance

\begin{abstract}
A l'instar de la plupart des pays africains, le Togo est à la recherche du modèle constitutionnel qui convienne à ses réalités politiques et socioculturelles. Dès les années I99o, considérées comme une période de renouveau démocratique, l'organisation constitutionnelle s'était orientée vers un régime de type parlementaire, appréhendé à tort ou à raison comme un instrument de limitation des pouvoirs du chef de l'Etat. Mais l'expérience malheureuse de ce genre d'organisation des pouvoirs politiques fait penser à un autre modèle constitutionnel d'inspiration présidentielle.

Mots clés: régime politique, régime parlementaire, régime présidentiel, chef de l'Etat, président de la république, premier ministre, contrepouvoir
\end{abstract}

\section{Introduction}

"L'Afrique n'a pas besoin d'homme fort, mais d'institutions fortes ", pouvait déclarer le Président américain, Barak Obama, au Parlement ghanéen le II juillet 2009. Si à première vue une telle déclaration constitue une invite aux peuples africains, aux gouvernants africains à faire foi aux institutions plutôt que de placer leur confiance aux hommes, elle contient tout de même au fond le constat d'une Afrique qui cherche toujours désespérément ses institutions et des hommes dignes de les représenter. 
De toute évidence, "l'Afrique cherche. L'Etat cherche ses institutions, la démocratie son expression, la justice son éthique, la société ses valeurs, l'économie sa croissance ; le peuple, lui, cherche sa survie $»^{1}$.

L'on a pu dire à juste titre que depuis son indépendance, l'Afrique "adopte, remet en cause, suspend, abroge, puis renouvelle la constitution ; elle cherche sa voie... $\prime^{2}$. Le Togo, ne saurait malheureusement échapper à ce constat d'une Afrique qui se cherche.

En effet, à l'instar de la plupart des pays d'Afrique noire francophone, au lendemain de son indépendance le 27 avril I960, au nom de la stabilité politique et de l'efficacité gouvernementale, les premiers dirigeants du Togo avaient substitué la constitution de type présidentiel à la constitution d'inspiration parlementaire héritée du colonisateur français ${ }^{3}$. Comme si cela ne suffisait pas, au nom du développement, de l'unité nationale, le monolithisme politique a été substitué au pluralisme politique en $1969^{4}$. Mais dès la fin des années I980, l'échec du parti unique en matière de développement et de la construction de l'unité nationale était patent. Le parti unique avait au contraire contribué à institutionnaliser les élections sans choix ou adhésion, la dictature, le pouvoir personnel, la patrimonialisation du pouvoir, la violation massive des droits de l'hommes.

A partir des années 1989 , avec la fin de la guerre froide, consécutive à la chute du mur de Berlin, à la dislocation de l'URSS, les régimes autoritaires allaient perdre le soutien cynique dont ils bénéficiaient de la part des puissances internationales ${ }^{6}$. Celles-ci commencèrent à les pousser à opérer des réformes démocratiques ${ }^{7}$. C'est ainsi qu'à l'instar des autres pays d'Afrique noire francophone, le Togo allait connaître dès les années I990, une mutation sociale, constitutionnelle et institutionnelle sans précédent. A cor et à cri, le peuple togolais avait exigé et obtenu l'abandon du régime à parti unique, auquel il attribuait toutes les tares du pays, au profit d'un système de Gouvernement plus libéral ou démocratique, porteur à tort ou à raison de beaucoup d'espoir. Le passage du

I Jean-Jacques Raynal, «Le renouveau démocratique béninois : modèle ou mirage ", Afrique contemporaine, $\mathrm{n}^{\circ}$ I6o, I99I, p. 25.

2 Maurice Ahanhanzo Glele, cité par Koffi AHADZI, « Les nouvelles tendances du constitutionnalisme africain : le cas des Etats d'Afrique noire francophone ", La revue du CERDIP, volume I, $\mathrm{N}^{\circ}$ 2, Juillet-décembre 2002, p. 35.

3 Cf.LouisDubouis, «LerégimeprésidentieldanslesnouvellesconstitutionsdesEtatsafricainsd'expressionfrançaise» Penant, ig62, p. 221 et suivant.

4 Pour avoir une vue générale sur la question, cf. Gérard Conac, «Les processus de démocratisation en Afrique », In Gérard Conac (dir), L'Afrique en transition vers le pluralisme politique, op. cit., p. I3 ; Dimitri Georges Lavroff, Les systèmes constitutionnels en Afrique noire : les Etats francophones, op. cit., pp. 73-78 ; Théodore Holo, « Démocratie revitalisée ou démocratie émasculée? Les constitutions du renouveau démocratique dans les Etats de l'espace francophone africain : régimes juridiques et systèmes politiques ", Revue béninoise des sciences juridiques et administratives, $n^{\circ} \mathrm{I} 6$, année 2006 , p. I7.

5 Cf. Gérard Conac, «Les processus de démocratisation en Afrique », in Gérard Conac (dir), L'Afrique en transition vers le pluralisme politique, op. cit., p. I6 ; Cf. Koffi Ahadzi, « Les nouvelles tendances du constitutionnalisme africain : le cas des Etats d'Afrique noire francophone", op. cit., p. 37.

6 Cf. Pierre-François Gonidec, « Démocratie et développement en Afrique : perspectives internationales et nationales ", Afrique 2000, Revue africaine de politique internationale, Trimestre ${ }^{\circ}{ }^{1} 4$ : juillet-août-septembre I993, pp. 5I-5I.

7 Cf. Koffi Ahadzi, "Les nouvelles tendances du constitutionnalisme africain : le cas des Etats d'Afrique noire francophone ", op. cit., p. 37 ; Théodore Holo, "Démocratie revitalisée ou démocratie émasculée ? Les constitutions du renouveau démocratique dans les Etats de l'espace francophone africain : régimes juridiques et systèmes politiques ", op. cit., p. I8. 
parti unique à un régime démocratique s'était fait au moyen de la conférence nationale ${ }^{8}$. Tenue de juillet à août I99I, celle-ci avait mis en place une commission constitutionnelle chargée de rédiger une nouvelle constitution. Le I4 octobre I992, après plusieurs mois de controverses au sein de la commission constitutionnelle sur la nature du régime po-

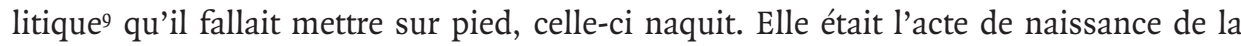
IVe République togolaise qui était censée mettre fin à la transition démocratique (I99II993 $)^{\mathrm{IO}}$ et instituer un régime politique à la fois durable ou stable et protecteur des droits et libertés des individus. Seulement, il semble que cette Constitution est handicapée, car elle peine à grandir, reste fragile et manque souvent de souffle. Peut-être c'est ce souffle qui est recherché à travers les interminables négociations entreprises par les acteurs politiques togolais depuis l'adoption de la constitution de 1992 et qui ont abouti à de multiples accords politiques, notamment Accords de Ouagadougou en I993, Accord-cadre de Lomé de I999, Accord politique global de 2006. Les échecs ainsi constatés sontils d'ordre institutionnel ? Sont-ils liés à la nature du régime dit semi-présidentiel à la française mis en place en I992 ? En tout cas, c'est dans ce sens que semblent pencher les acteurs politiques togolais, puisque l'Accord politique global de 2006 prévoyait les réformes d'ordre constitutionnel et institutionnel, notamment la redéfinition d'un nouveau régime politique. De même, si l'on admet de façon optimiste que toute révision constitutionnelle vise à améliorer les dispositions de la loi fondamentale, la révision constitu-

Selon le Professeur Koffi Ahadzi, «la conférence nationale peut se définir comme un forum réunissant toutes les forces vives de la Nation (syndicats, associations diverses, forces traditionnelles...) autour des représentants du pouvoir d'Etat aux fins de débattre des problèmes politiques, économiques et sociaux qui se posent à la communauté en vue d’y apporter des solutions. Selon que celle-ci a une portée obligatoire ou indicative vis-à-vis du régime en place, la conférence est dite souveraine ou non souveraine "; Koffi Ahadzi, «Les nouvelles tendances du constitutionnalisme africain », op. cit. p. 37, note II.

9 Entendue généralement comme le mode de Gouvernement auquel est soumis un pays, la notion de régime politique renvoie à une réalité complexe à cerner. Selon Georges Burdeau, le régime politique peut être légitimement assimilé à l'étude des formes constitutionnelles, des règles d'organisation et de fonctionnement des pouvoirs publics. Ainsi, en distinguant « les divers procédés selon lesquels sont désignés les gouvernants, en comparant les compétences dont ils sont investis, en classant les différentes manières selon lesquelles ils remplissent leur fonction, on pourrait rendre compte des particularités de chaque forme gouvernementale et en dégager un schéma théorique susceptible de servir de base à une classification des régimes ". Mais il faut convenir volontiers que la notion du régime politique ne saurait être réductible à la description des formes gouvernementales, elle doit aussi prendre nécessairement en compte la philosophie sociale, c'est-à-dire " la manière dont la doctrine gouvernementale conçoit la valeur et le rôle de l'individu, l'orientation qu'elle tend à donner aux relations sociales, les fins qu'elle assigne aux activités individuelles.... ". Ces deux visions du phénomène, loin de s'exclure se complètent plutôt. En effet, compte tenu du fait que la constitution ordonne une forme particulière de Gouvernement autour d'une philosophie sociale spécifiquement appropriée aux fins assignées aux pouvoirs publics, certains auteurs ont proposé justement une définition synthétique du régime politique appréhendée comme étant " l'ensemble des règles constitutionnelles "; cf. Olivier Duhamel Yves Meny, Dictionnaire Constitutionnel, PUF 1992, p. 887 ; Georges Burdeau, Traité de Sciences Politique, les régimes politiques, ze édition, tome V, L.G.D.J, Paris, I970 pp. 2-I4 Georges Burdeau, Traité de Sciences Politique, les régimes politiques, 2e édition, tome V, L.G.D.J, Paris, I970 pp. 2-I4 ; Marie-Anne Cohendet, Droit Constitutionnel, 2e édition, Montchrestien, EJA, 2002 p. 3 I2.

Io Après un soulèvement populaire dans les années I990, le Togo a connu une période de transition (I99I-I993), marquée par la suspension de la Constitution de 1980 et des institutions qui en étaient issues. Durant cette période, la conférence nationale dite souveraine avait mis en place un Haut conseil de la République (H.C.R.), qui faisait office du pouvoir législatif, et un Gouvernement provisoire à la tête duquel se trouvait un premier ministre. 
tionnelle de 2002 pourra être analysée comme étant un moyen pour donner un nouveau souffle à la Constitution de $1992^{\text {II }}$. Il ressort donc que la constitution togolaise de 1992 semble connaître ses limites, auxquelles les acteurs politiques ont répondu soit par des négociations politiques, soit par la voie de révision constitutionnelle. Les réponses apportées par ceux-ci en vue de redynamiser ou de booster la constitution de 1992 ont-elles eu des effets escomptés? Une réponse négative nous caresse légitimement l'esprit, car nous devons considérer humblement que les belles architectures, annonciatrices d'une ère nouvelle n'ont pas pu tenir leur serment et le constat reste amer aussi bien pour les gouvernants que pour les gouvernés. Si l'on suppose que ces échecs, ces lacunes, ces insuffisances sont imputables à la nature du régime politique togolais actuel, alors quel régime politique pour le Togo ? Lequel des régimes parlementaire et présidentiel convient le mieux à l'environnement politique togolais actuel ?

La question n'est pas si aisée à répondre, car sur le plan scientifique tous les régimes politiques se valent, et politiquement la question est si sensible. La prudence doit alors être de mise, car toute prise de position pourrait être interprétée en fonction des intérêts personnels, égoïstes et divergents des protagonistes. C'est pourquoi la réponse de cette question se veut être empirique. En toute évidence, en observant la vie politique togolaise depuis 1992, il ressort que l'expérience du régime d'inspiration parlementaire a été malheureuse (I), ce qui fait songer à l'expérimentation du régime présidentiel (II).

\section{L'expérience malheureuse du régime d'inspiration parlementaire}

Le régime parlementaire désigne "le régime politique au sein duquel les pouvoirs publics collaborent et dépendent l'un de l'autre, autrement dit, la vie de ménage $»^{{ }^{2}}$. Dans le même sens, les Professeurs Philippe ARDANT et Bertrand MATHIEU le définissent comme un régime politique "caractérisé par la collaboration de l'exécutif et du législatif, le premier étant indépendant mais responsable devant le second "13. Dans un tel régime, le Gouvernement doit disposer, pour son maintien et sa survie, à tout moyen de la confiance de la majorité parlementaire. Il renvoie aussi à une forme d'organisation du pouvoir exécutif marquée par l'existence d'un chef de l'Etat ou d'un monarque, qui ne joue pas un rôle politique actif et d'un Premier ministre, chef du Gouvernement, qui émane de la majorité parlementaire et exerçant l'essentiel du pouvoir exécutif. Mais cette forme d'organisation du pouvoir exécutif présente des inconvénients sérieux : le dualisme de l'exécutif risque à tout moment de provoquer une crise profonde au sommet de l'Etat surtout dans le contexte africain, car le duo qui suppose compromis sur les questions essentielles, se changera très vite en un duel permanent et inexpugnable jusqu'à la disparition de l'un des acteurs ${ }^{\text {I4 }}$. C'est ce que

\footnotetext{
II La constitution togolaise de 1992 a été profondément révisée par la loi n²002-029 du 3I décembre 2002 par une Assemblée nationale presque monocolore, composée de 72 députés du Rassemblement du peuple togolais (RPT), parti au pouvoir, sur les 8I que compose l'Assemblée nationale togolaise.

I2 Olivier Duhamel et Yves Meny, Dictionnaire constitutionnel, PUF I992, p. 883.

I3 Philippe Ardant et Bertrand Mathieu, Institutions Politiques et Droit Constitutionnel, 22e édition, L.G.D.J, 20Io, p. 232.

I4 Cf. Philippe Manga, " Réflexions sur la dynamique constitutionnelle en Afrique », R.J.P.I.C, I994, Janvier-Avril $\mathrm{N}^{\circ}$ I, p. 66 .
} 
l'on a pu constater lors de la période de transition (I99I-I993) (A) et lors de la cohabitation Eyadema-Kodjo (B).

\section{Les crises liées au dualisme de l'exécutif lors de la transition}

Lors de la période de transition au Togo, l'organisation et le fonctionnement des pouvoirs publics étaient l'œuvre d'un acte adopté le 23 août I99I par la Conférence nationale dite souveraine, dénommé l'Acte fondamental $n^{\circ} 7$. Ce dernier prévoyait un exécutif dualiste : le Président de la République d'alors critiqué, Gnassingbé Eyadema, était maintenu dans ses fonction $\mathbf{s}^{15}$, mais ses pouvoirs étaient sérieusement réduits au profit du premier ministre de la transition, Me Joseph Kokou Koffigoh, qui exerçait l'essentiel du pouvoir exécutif.

En effet, aux termes de l'acte $n^{\circ} 7$ portant Loi constitutionnelle, le Premier ministre de transition togolaise, Joseph Kokou Koffigoh, disposait d'importantes attributions. Selon l'article 35 de la Loi constitutionnelle, "le Premier ministre préside le conseil des ministres". Il exerçait à titre exclusif le pouvoir réglementaire, puisque l'article 36 disposait que «le Premier ministre signe les décrets délibérés en conseil des ministres». Il nommait aux emplois civils et militaires de l'Etat dans la mesure où « les membres de la Cour suprême, le Grand Chancelier de l'Ordre du Mono, les Ambassadeurs et envoyés extraordinaires, les préfets, les officiers généraux, le président de l'Université du Bénin élu par ses pairs, les Directeurs des administrations Centrales sont nommés en conseil des ministres après avis du bureau du Haut conseil de la République...». Il ressort donc de cette disposition que le Premier ministre et son Gouvernement avaient non seulement le monopole du pouvoir réglementaire, mais aussi et surtout celui du pouvoir de nomination dans la mesure où l'avis du bureau du Haut Conseil de la République était juridiquement un avis simple qui est loin de lier le Premier ministre. Dans le même sens, le Premier ministre n'avait pas " de comptes à rendre au Président de la République qui était seulement " informé des activités du Gouvernement $»^{16}$.

Contrairement à l'avis de certains qui prônaient une destitution pure et simple des chefs d'Etat pendant la période de transition, ces derniers furent maintenus à leur poste. Mais l'institution présidentielle était apparue comme " une institution passive " ${ }^{\mathrm{T}}$. Aux termes de cet acte fondamental, le chef de l'Etat devait jouer " un rôle essentiellement honorifique comme dans un régime parlementaire classique, un rôle tout au plus contemplatif, sinon décoratif ${ }^{18}$.

En effet, seuls les pouvoirs traditionnellement confiés au chef de l'Etat de type parlementaire classique étaient conservés au chef de l'Etat, Gnassingbé Eyadema. Il " incarnait la continuité de l'Etat, l'indépendance et l'unité nationale. Il assurait le respect des traités et accords

I5 L'acte $\mathrm{n}^{\circ} 3$ adopté par la conférence nationale le 9 août I99I disposait que "le Président de la République est provisoirement maintenu dans ses fonctions protocolaires de chef de l'Etat sous le contrôle de la conférence nationale (...) ".

I6 L'article 40 de l'acte $n^{\circ} 7$ portant Loi constitutionnelle de la période de transition au Togo disposait que "le Premier ministre tient le Président de la République informé des activités du Gouvernement ".

I7 Aboubacar Maïdoka, "La nouvelle organisation des pouvoirs publics nigériens : L'acte fondamental $n^{\circ} 2 \mathrm{I}$ ", R.J.P.I.C., n $^{\circ}$, janvier-mars 1992, p. 48.

I8 Anani Kaleto Edem Koudouovoh, "Le régime politique de la période de transition au Togo ", Mémoire pour l'obtention de la maîtrise Es-Science juridique, Option : carrière administrative, Université de Lomé, Année universitaire I99I-I992, p. I8. 
internationaux. Il [était] chef suprême des armées. Il représentait l'Etat à l'étranger "19. En outre, aux termes de l'article 29 de l'acte $n^{\circ} 7$, le Président de la République accréditait les Ambassadeurs et les envoyés extraordinaires de la République togolaise auprès des puissances étrangères. De même, les Ambassadeurs et les envoyés extraordinaires étrangers étaient accrédités auprès de lui. Il faut noter que ces pouvoirs ont un contenu incertain et ne faisaient du chef de l'Etat qu'un symbole vivant. Il exerçait le droit de grâce et recourait au référendum constitutionnel sur "proposition du Premier ministre ". De plus, non seulement le chef de l'Etat devait promulguer "les lois dans les 8 jours qui suivent leur transmission au Gouvernement ", mais aussi l'article 6o neutralisait son éventuel pouvoir de résistance, car "les lois régulièrement votées par le Haut Conseil de la République et non promulguées par le Président de la République dans le délai prévu, le sont valablement par le Premier ministre ".

Mais cette organisation des pouvoirs de transition prévue par l'acte $n^{\circ} 7$ n'avait pas eu la chance de fonctionner, car " la cohabitation à la togolaise avait vécu avant même de naître [et le Premier ministre Koffigoh était] pris en otage et [ne pouvait] rien faire face à la menace des armes $\|^{20}$. En effet, le 28 novembre I99I, les militaires avaient réussi à orchestrer un coup de force contre la primature. Ils avaient pris en otage le Premier ministre pendant une semaine. Celui-ci finit par se rendre au Président de la République. Le Premier ministre, Joseph Kokou Koffigoh avait annoncé le 3 décembre I99I à la télévision et à la radio le gel du programme de transition, la suspension des institutions mises en place par la conférence nationale et de la légalité républicaine et avait consenti à la formation d'un Gouvernement provisoire d'union nationale, et ce " afin d'éviter un bain de sang ". Ce coup porté aux institutions de la transition a permis au chef de l'Etat de reconquérir ses pouvoirs et d'asseoir son autorité sur les autres organes de transition.

En effet, à la suite de ce coup de force, le Président du H.C.R., Mgr Fanokpo Kpodzro exprimait la subordination de son institution en ces termes : "le H.C.R. est descendu de son piédestal d'organe suprême (...). Nous avons assoupli nos méthodes. Nous avons abandonnéla méthode de Mars pour adopter la méthode de Venus $»^{21}$. Aussi, la formation d'un Gouvernement d'union nationale en concertation avec le chef de l'Etat avait eu pour inconvénient de réduire la marge de manœuvre du Premier ministre qui devait désormais négocier le pouvoir dans certains domaines avec le chef de l'Etat. Cette subordination du Premier ministre et du H.C.R. au Président de la République deviendra définitive avec la modification de l'acte $\mathrm{n}^{\circ} 7$ consécutive aux négociations de la commission paritaire qui avait réuni du 28 juillet au i9 août 1992 la mouvance présidentielle et l'opposition ${ }^{22}$. Ces négociations avaient conduit à la modification de l'article 26 de l'acte fondamental, modification qui autorisait le chef de l'Etat à présider le conseil des ministres « en fonction de l'importance des sujets inscrits à l'ordre du jour ". A partir de cette modification, Gnassingbé Eyadema avait présidé tous les conseils

I9 Article 26 de l'acte fondamental $n^{\circ} 7$.

20 Elimane Fall, " Koffigo : la plus haute des solitudes », Jeune Afrique, nㄷ6I5, II au I7 décembre I99I, p. I5.

2I Voir Anani Kaleto Edem Koudouovoh, op. cit, p. 33.

22 Voir dans ce sens, Géraldine Faes, "Comment Eyadéma a reconquis le pouvoir ", J.A., n655, 24 au 30 septembre 1992 ; Jean du Bois de Gaudusson, Gérard Conac et Christine Desouches, Les constitutions africaines publiées en langue française, tome 2, op. cit, p. 373. 
des ministres quel qu'en soit l'ordre du jour. Aussi, la modification de l'article 36 n'étaitelle pas moins importante quant à la marge de manœuvre du Premier ministre. Aux termes de cette modification, ce dernier formait le Gouvernement " en attente avec le Président de la République", d'une part, et les décrets de nomination des membres du Gouvernement étaient signés par "le Président de la République » et le Premier ministre, d'autre part. Toutes ces circonstances avaient permis au chef de l'Etat de contrôler l'exécutif et de conduire à son profit la période de transition chaotique. Le même duel a eu lieu, après la période de transition, entre l'ex-Président de la République Eyadema et l'ancien PM Edem Kodjo.

\section{La cohabitation conflictuelle entre Eyadema et Kodjo}

La Constitution togolaise de I992, calquée sur la Constitution française de 1958, favorise une cohabitation à la tête de l'exécutif23. Ainsi, les élections législatives de I994, remportées par l'opposition ${ }^{24}$, avaient conduit à la cohabitation entre le Président de la République d'alors, Gnassingbé Eyadema et l'ex-premier ministre, Edem Kodjo. Cette cohabitation a été conflictuelle surtout sur le partage des pouvoirs de nomination et sur les pouvoirs attachés à la présidence du conseil des ministres que la Constitution de I992 reconnaît au chef de l'Etat.

En effet, alors qu'en juin I995, le Premier ministre estimait que " la cohabitation avec le Président Eyadema se déroule plutôt bien $»^{25}$, en avril 1996 , elle se transformera en un duel remporté sans doute par le chef de l'Etat. Après s'être confié à Dieu plus d'un $a^{26}$, le Premier ministre tenta dès avril 1996 de réagir contre la marginalisation dans laquelle le Président Eyadema le plongeait. Ce qui fut à l'origine de l'épreuve de force entre les deux chefs de l'exécutif, c'était la nomination des hauts fonctionnaires ${ }^{27}$. Tout commença par une lettre circulaire du I7 avril I996 adressée par le Président Eyadema aux membres du Gouvernement. Dans cette lettre, il rappela aux ministres qu'en vertu de l'article 70 de la constitution $^{28}$, la nomination des directeurs de l'administration centrale lui revenait, et

23 La cohabitation est entendue comme la période au cours de laquelle le parti politique du Président de la République ne dispose pas d'une majorité à l'assemblée nationale. Il est alors obligé de nommer un Premier ministre issu d'un parti de l'opposition avec lequel il doit composer.

24 Les résultats des législatives des 6 et 27 février 1994 donnèrent une majorité à l'opposition qui remporta 40 sièges sur les 8r à pourvoir, dès lors que l'élection de trois (3) députés a été invalidée. En effet, après l'annulation de l'élection de 3 députés de l'opposition, le Comité d'action pour le renouveau (CAR) de Yaovi Agboyibo obtint 34 sièges et l'Union togolaise pour la démocratie (UTD) d'Edem Kodjo 6 sièges. Par contre, le Rassemblement du peuple togolais (RPT) du Président Eyadéma, ancien parti unique et parti au pouvoir, obtint 35 sièges. Le RPT pouvait aussi compter sur les 2 sièges de l'Union pour la justice et la démocratie (UJD) et celui de la Confédération des forces nouvelles (CFN), qui lui étaient affiliées. Cette configuration de l'Assemblée nationale obligeait le chef de l'Etat à nommer le Premier dans les rangs de l'opposition. Kodjo : « un an avec Eyadéma », Jeune Afrique n'고 du 25 au 3I mai I995, p. 56.

Selon l'un des proches du Premier ministre, « tous les matins que Dieu fait, à sept heures, Edem Kodjo se rend à la messe. La foi l'aide à tenir ", voir François SOUDAN, " Eyadéma-Kodjo, jusqu’à quand ? ", Jeune Afrique n ${ }^{\circ}$ 8I 5 du ig au 25 octobre i995, p. 38.

Voir Hamza Kaïdi, «Les lettres de la discorde », Jeune Afrique n 1852 du 3 au 9 juillet, p. 27.

L'article 70 de la constitution togolaise du I4 octobre I992, version originale, disposait que « le Président de la République, après délibération du conseil des ministres, nomme le grand chancelier de l'Ordre du Mono, les ambassadeurs et envoyés extraordinaires, les préfets, les officiers commandants des armées de terre, de mer et 
par conséquent, les nominations qui étaient intervenues en violation de cette disposition étaient nulles et de nul effet et devaient être immédiatement rapportées. Le même jour, il adressa une lettre au Premier ministre dans laquelle il rappela ces mêmes dispositions. Il y précisa que les dispositions de l'article 70 s'appliquaient à tous les fonctionnaires d'un rang égal ou supérieur à celui de directeur de l'administration centrale, donc aux secrétaires généraux de la primature et des ministères, ainsi qu'aux directeurs d'administration centrale. Il ajouta plus loin qu'en sa qualité du Président du conseil des ministres, il lui appartenait d'arrêter l'ordre du jour, de formuler et de formaliser les décisions prises après délibération.

En réponse à la lettre du chef de l'Etat, le Premier ministre adressa une longue lettre à ce dernier le 22 avril i996. Dans cette correspondance, il rappela au chef de l'Etat que la question des nominations n'était pas réglée dans son principe par l'article 70 de la constitution mais par l'article 79 de la même constitution ${ }^{29}$. Au sujet des pouvoirs attachés à la présidence du conseil des ministres, il écrit que "s'il est exact que le Président de la République, en sa qualité du Président du conseil des ministres, arrête l'ordre du jour du conseil, il appartient au chef de Gouvernement d'en établir le projet conformément à l'article 77 de la constitution qui veut que le Gouvernement détermine et conduise la politique de la nation $13^{30}$. Pour finir, le Premier ministre saisit l'occasion pour exprimer son ras-le-bol face à l'exclusion politique dont il a été victime de la part du Président Eyadema ${ }^{\text {II }}$. Le 23 avril I996, Edem Kodjo adressa au Président Eyadema une nouvelle lettre bien plus sèche : "J'ai le regret de vous informer que votre circulaire en date du 17 avril 1996, adressée aux membres du Gouvernement, est irrecevable dans sa forme, dans la mesure où vous n'êtes pas le chefdu Gouvernement. Si vous avez des observations à formuler sur les questions évoquées par votre lettre circulaire, vous devez les adresser au chef du Gouvernement qui se chargera d'en répercuter le contenu aux ministres.... ". Face à cette interprétation divergente des articles 70, 77 et même

de l'air et les directeurs des administrations centrales. Le Président de la République, par décret pris en conseil des ministres, nomme les Présidents d'universités élus par les collèges électoraux des universités, les Professeurs inscrits sur une liste d'aptitude reconnue par les conseils des Universités et les officiers généraux. Une loi organique détermine les autres emplois auxquels il est pourvu en conseil des ministres ainsi que les conditions dans lesquelles le pouvoir de nomination du Président de la République peut être par lui délégué pour être exercé en son nom ".

29 Article 79 de la constitution du I4 octobre I992, version originale : « Le Premier ministre assure l'exécution des lois. Sous réserve des dispositions de l'article 7o, le Premier ministre nomme aux emplois civils et militaires. Il peut déléguer certains de ses pouvoirs aux ministres ».

Article 77 de la constitution du I4 octobre I992, version originale : «Le Gouvernement détermine et conduit la politique de la nation. Il dirige l'administration civile et militaire. A cet effet, il dispose de l'administration, de la force armée et des forces de sécurité. Le Gouvernement est responsable devant l'Assemblée nationale ".

Le Premier ministre exprime ainsi son ras-le-bol : « Vous reconnaîtrez que j'ai fait preuve d'une grande souplesse dans la revendication de mes droits constitutionnels et dans leur mise en œuvre. A quelle nomination militaire ai-je procédé depuis mon entrée en fonction ? (...) Peut-on considérer que le Gouvernement détermine et conduit la politique de la nation en matière de politique étrangère alors que les missions sont conduites à l'étranger sans que j'en sois informé ? " Faisant allusion aux discussions bénino-togolaises lors de visite du Président Kérékou à Kara, Edem Kodjo écrit au Président Eyadéma : « Peut-on considérer que le Premier ministre a son mot à dire dans le domaine des relations internationales lorsqu'il passe quatre heures dans une chambre à Kara sans jamais avoir été associé, ne serait-ce qu'épisodiquement, aux négociations ? Même le communiqué final ne m’a pas été montré "; voir Jeune Afrique n¹ 852 , op. cit. pp. 27-28. 
de l'article 66 sur la présidence du conseil des ministres, le Président Eyadema saisit la Chambre constitutionnelle de la Cour Suprême d'une demande d'avis portant d'abord sur les pouvoirs attachés à la présidence du conseil des ministres, ensuite sur la notion de la majorité parlementaire et enfin sur les pouvoirs respectifs du Président de la République et du Premier ministre en matière de la nomination des hauts fonctionnaires.

Concernant le premier point, dans son avis du 4 juin I996, la Cour estima que la présidence du conseil des ministres signifie que c'est le Président de la République qui " arrête définitivement l'ordre du jour du conseil, c'est lui qui décide des sujets à débattre. C'est lui qui dirige les débats et oriente les travaux (...) et conclut les discussions. La présidence du conseil des ministres est une fonction effective qui conferre au Président de la République des pouvoirs réels dans la détermination de la politique de la nation; le Gouvernement la conduit conformément aux orientations du chef de l'Etat, sous le contrôle du Parlement " $3^{2}$. Sur ce point, la Cour conclut que "la constitution, en confiant au Président de la République la présidence du conseil des ministres, entend lui conférer ainsi la prééminence sur le Premier ministre ". A propos du troisième point, la Chambre constitutionnelle n'avait pas fait mieux. Elle estima que "le pouvoir de nomination du Premier ministre est un pouvoir résiduel qui s'étend aux domaines non couverts par l'article 70 de la constitution et la loi organique à laquelle renvoie cet article ${ }^{33}$.

Cette situation conflictuelle avait conduit à un malaise institutionnel. En l'absence d'une entente entre le chef de l'Etat et le Premier ministre, le fonctionnement des pouvoirs publics avait pris un coup : les conseils des ministres étaient rarement convoqués, les nominations de hauts fonctionnaires furent bloquées ou opérées unilatéralement, la mise en place des institutions prévues par la constitution du I4 octobre I992 fut paralysée ${ }^{34}$. Beaucoup d'observateurs de la politique africaine trouvent que la cohabitation est particulièrement périlleuse puisqu'elle conduit " à l'affrontement entre le chef de l'Etat et le Premier ministre et donc au blocage ${ }^{35}$ des institutions. Ils concluent que le modèle constitutionnel à la française, "qui expose à plus de dangers que de bienfaits, est à écarter $»^{6}$ en Afrique, et expriment leur préférence pour le régime présidentiel.

Avis de la Chambre constitutionnelle de la Cour Suprême du 4 juin 1996 sur l'interprétation des articles 66 et 70 de la constitution togolaise du I4 octobre 1992.

33 Quant au 2e point, la Cour estima qu'on entend par majorité parlementaire, «le parti ou la coalition de partis détenant la majorité de sièges au Parlement (moitié plus un) et servant d'appui au Gouvernement. Cette majorité est fluctuante (au gré des questions débattues). C'est au moment des votes qu'il se constitue une majorité conjoncturelle, car les députés ne sont pas liés par un mandat impératif".

34 Ahadzi-Nonon Koffi, « Le Premier ministre en Afrique noire francophone : étude de quelques exemples récents ", Revue nigérienne de droit, Revue semestrielle nºr novembre 1999, p. 7I.

35 Koffi Ahadzi, "Les nouvelles tendances du constitutionnalisme africain : le cas des Etats d'Afrique noire francophone ", op. cit. p. 77 .

36 Philippe Manga, "Réflexion sur la dynamique constitutionnelle en Afrique ", op.cit., p. 66. 


\section{L'expérimentation du régime présidentiel}

Instruit par l'échec du bicéphalisme en Afrique noire francophone ${ }^{37}$ en général et plus particulièrement au Togo, et le succès relatif du monocéphalisme au Bénin ${ }^{38}$, il est logique que l'on se prononce en faveur de cette dernière forme d'organisation du pouvoir exécutif qui " confond dans la personne de son titulaire les fonctions de chef de l'Etat et de chef du Gouvernement "39. Elle serait un gage de stabilité et d'efficacité gouvernementales. Mais la prudence invite également à ressortir, comme aux Etats-Unis, les pouvoirs présidentiels de freins et de contrepoids afin d'éviter le retour facile au pouvoir personnel. Ainsi, il s'agira de confier la totalité du pouvoir exécutif au Président de la République (A) et de renforcer les organes de contrepouvoirs (B).

\section{Confier la totalité du pouvoir exécutif au Président de la République}

Aux Etats-Unis d'Amérique, le Président de la République est le chef incontesté de l'exécutif. Selon Theodore Roosevelt, il cumule "l'autorité d'un roi et d'un Premier ministre »40. En prenant à notre compte cet aménagement institutionnel, il paraît souhaitable que le Président de la République soit à la fois chef de l'Etat et chef du Gouvernement. Ainsi le poste de Premier ministre pourra être supprimé.

Cependant, compte tenu de l'importance des tâches gouvernementales, le chef de l'Etat pourra nommer à sa guise un Premier ministre révocable par lui à tout moment. Sinon, comme le disait Edward Corwin, la présidence serait un "killing job " ${ }^{41}$. Ce dernier sera chargé de coordonner l'action du Gouvernement. "Ce bras-droit administratif aura pour mission de décharger [le chef de l'Etat] des tâches quotidiennes d'exécution, ce qui lui permettra de se consacrer à la définition des grandes orientations nationales ${ }^{42}$. Le Premier ministre ainsi nommé formera un duo avec le Président de la République, car il n'existera "pas deux têtes, deux acteurs qui pèsent d'un poids égal, mais un seul sur qui repose la direction de l'Etat. Cela suppose qu'entre les deux, il n'existe pas de zones de compétences réservées, mais que l'un assume la plénitude du pouvoir exécutif tandis que l'autre ne représente qu'une ombre fugitive "43. Par conséquent, les risques d'une cohabitation à la française seront définitivement exclus. Ce qui sera un gage "de stabilité, d'efficacité et de rapidité "44 de l'action gouvernementale.

En sa qualité de chef du Gouvernement, le Président de la République devra être logiquement maître de la composition du Gouvernement. Ainsi, il devra non seulement

37 Voir Philippe Manga, "Réflexion sur la dynamique constitutionnelle en Afrique ", op.cit., p. 65 et s. ; Alioune SALL, "Processus démocratique et bicéphalisme du pouvoir exécutif en Afrique noire francophone: un essai de bilan ", op. cit.

38 Voir Koffi Ahadzi "Les nouvelles tendances du constitutionnalisme africain : le cas des Etats d'Afrique noire francophone ", op. cit., p. 77.

39 Jean Gicquel et Jean-Eric Gicquel, Droit Constitutionnel et institutions politiques, op. cit., p. 279.

40 Idem.

4I Cité par Jean Gicquel et Jean-Eric Gicquel, Droit Constitutionnel et institutions politiques, op. cit., p. 295.

42 Koffi Ahadzi, "Les nouvelles tendances du constitutionnalisme africain : le cas des pays d'Afrique noire francophone ", op. cit., p. 84 .

43 Philippe Manga, "Réflexion sur la dynamique constitutionnelle en Afrique ", op. cit., p. 66.

44 Koffi Ahadzi, "Les nouvelles tendances du constitutionnalisme africain : le cas des pays d'Afrique noire francophone ", op. cit., p. 84 . 
nommer les ministres, mais aussi fixer leurs attributions et mettre fin à leurs fonctions. Certes, comme dans la plupart des pays d'Afrique francophone, au Togo, le Président de la République dispose en pratique du pouvoir de révocation des membres du Gouvernement. Mais, comme en France, ce pouvoir de révocation reste encore dépendant des circonstances politiques : en cas de convergence entre majorités présidentielle et parlementaire, il dispose du pouvoir de révocation des ministres, mais en cas de divergence de majorités, il perd ce pouvoir. C'est pourquoi, il faudra que le Président de la République détienne la liberté de révocation des ministres du texte de la constitution. "Puisque le Président doit faire une politique, il doit être libre de choisir les instruments de cette politique "45 d'une part, et libre de les changer en cas de besoin, d'autre part. Toujours en sa qualité de chef du Gouvernement, le chef de l'Etat devra définir et conduire la politique de la nation. Actuellement, c'est sous l'autorité du chef de l'Etat que le Gouvernement définit et conduit la politique de la nation, ce qui constitue une poudrière en cas de cohabitation. Pour éviter tout risque de conflit au sommet de l'Etat, il faudra que le chef de l'Etat définisse et détermine la politique de la nation. Cette mission sera conforme à son mode de désignation, car "ce Président élu par le peuple est choisi pour faire une politique et c'est cette politique qu'il va imposer au pays $»^{4}$. Il doit également disposer d'un pouvoir réglementaire et de nomination qui lui permettra d'appliquer sa politique. Mais il faudra voir comment certains de ces pouvoirs de nomination, surtout la nomination des directeurs généraux des administrations centrales, pourront être partagés avec le Parlement, comme cela se fait aux Etats-Unis d'Amérique.

Cette organisation du pouvoir exécutif aura le mérite d'éviter des crises au sommet du pouvoir exécutif et de favoriser l'efficacité et la stabilité du Gouvernement. Mais elle présente aussi l'inconvénient de favoriser le pouvoir personnel. C'est pourquoi, sa mise en œuvre suppose certains préalables : le renforcement des organes de contrepouvoir et la promotion de la culture démocratique. Ainsi, afin d'éviter toute dérive autoritaire du pouvoir présidentiel liée à la nature présidentielle du régime, il faut chercher à encadrer les pouvoirs du chef de l'Etat. L'objectif recherché ici n'est pas d'empêcher le Président de la République d'exercer ses pouvoirs, mais au contraire, de rationnaliser l'exercice du pouvoir présidentiel.

\section{Renforcer les organes de contrepouvoir}

Convaincue, à l'instar des révolutionnaires français de 1789 , que " toute société dans laquelle la garantie des droits n'est pas assurée, ni la séparation des pouvoirs déterminée, n'a point de constitution $»^{47}$, la nouvelle élite sociale togolaise, à l'instar de ses homologues africaines, a multiplié, dès les années I990, les mécanismes de contrepouvoir ${ }^{48}$ en vue de créer un réel 
Etat de droit. Celui-ci se définit, selon Raymond Carré DE MALBERG, comme « un Etat qui, dans ses rapports avec ses sujets et pour la garantie de leur statut individuel, se soumet lui-même à un régime de droit, et cela en tant qu'il enchaîne son action sur eux par des règles, dont les unes déterminent les droits réservés aux citoyens, dont les autres fixent par avance les voies et moyens qui pourront être employés en vue de réaliser les buts étatiques : deux sortes de règles qui ont pour effet commun de limiter la puissance de l'Etat, en le subordonnant à l'ordre juridique qu'elles consacrent $"^{49}$. Les organes de contrepouvoir devront servir donc à assurer, d'une part, la suprématie et l'effectivité de la constitution et, d'autre part, le respect des droits et libertés fondamentaux consacrés et garantis par les nouvelles constitutions ${ }^{50}$. Pour atteindre ce double objectif, à côté des organes juridictionnels créés ou rénovés pour les besoins de la cause, des autorités administratives indépendantes ont vu le jour pour réguler un certain nombre de secteurs jugés sensibles ou essentiels. Ainsi, la création ou la revalorisation des organes juridictionnels s'imposait pour assumer "une fonction de régulation, lorsque le législateur se trompe et une fonction de censure si une majorité abuse de son Autorité "15. Dans ce sens, le nouveau constituant togolais, à l'instar de ses homologues de la sous-région, a pris le soin de créer une juridiction constitutionnelle autonome, chargée du contrôle de constitutionnalité de la loi, d'une part, et de réguler le fonctionnement des institutions et de l'activité des pouvoirs publics, et de garantir le respect des droits de l'homme et des libertés fondamentaux, d'autre part ${ }^{52}$. Dans la conscience collective africaine, la mise en place des juridictions constitutionnelles et des autorités administratives indépendantes devra servir de rempart contre les velléités autoritaires du chef de l'Etat, quand on sait que les empiètements spectaculaires sur les compétences des autres pouvoirs (exécutif et judiciaire), ainsi que les violations atroces des droits et libertés fondamentaux avant les années I990 étaient l'œuvre du Président de la République. Mais après vingt ans de mise en œuvre de ces institutions, les résultats sont encore largement en deçà des attentes, d'où la nécessité de renforcer le statut des organes juridictionnels et celui des autorités administratives indépendantes.

Le juge américain, Hugues, pouvait déclarer en I908 que « la constitution des Etats-Unis est ce que la Cour dit qu'elle est "53. Au regard de l'importance unanimement et universellement reconnue à la justice constitutionnelle, il s'avère nécessaire d'entourer la désignation de ses membres d'assez de précautions.

Au Togo, jusqu'en 2002, la Cour constitutionnelle togolaise était composée de sept membres dont deux étaient élus par l'Assemblée nationale, un désigné par le Président de

Soucramanien, Droit constitutionnel, op. cit., p. I4.

49 Raymond Carré de Malberg, Contribution à la théorie générale de l'Etat, tome I, op.cit., pp. 488-489; voir également Jacques Chevallier, "L'Etat de droit », Revue de droit public, I988, pp. 329 et s.

50 Voir Théodore Holo, "Démocratie revitalisée ou démocratie émasculée? Les constitutions du renouveau démocratique dans les Etats de l'espace francophone africain : régimes juridiques et systèmes politiques ", op. cit., pp. 19-3I ; Gérard Conac, "Quelques réflexions sur le nouveau constitutionnalisme africain ", op. cit.; Koffi Ahadzi, "Les nouvelles tendances du constitutionnalisme africain : le cas des Etats d'Afrique noire francophone ", op. cit., pp. 40-59.

5I R. Badinter, cité par Jean Gicquel et Jean-Eric Gicquel, Droit Constitutionnel et institutions politiques, op. cit., p. 755.

52 Article 99 de la Constitution togolaise de 1992.

53 Cité par Jean Paul Jacque, Droit constitutionnel et institutions politiques, op. cit., p. 77. 
la République, un par le Premier ministre et un magistrat, un avocat et un enseignant de la faculté de droit, élus par leurs pairs ${ }^{54}$. Quant au Président de la Cour constitutionnelle, il était élu par ses pairs ${ }^{55}$. Mais la révision constitutionnelle de décembre 2002 a modifié cette procédure de désignation en confiant seulement au Président de la République et au Parlement le pouvoir de désignation des membres de la Cour constitutionnelle. A l'issue de cette révision, la Cour constitutionnelle est désormais composée de neuf membres. En effet, le Président de la République, l'Assemblée nationale et le Sénat désignent chacun trois de ceux-ci dont un, dans chaque cas, en raison de ses compétences juridiques ${ }^{56}$. En attendant l'installation du Sénat, son pouvoir de désignation des trois membres de la Cour est actuellement confié à l'Assemblée nationale. Quant au Président de ladite Cour, il est désormais nommé par le Président de la République.

Le fait de confier la désignation des membres de la Cour constitutionnelle au Président de la République et au Parlement n'est pas à notre avis un gage d'indépendance de la Haute juridiction constitutionnelle, car les risques de récupération politique de celle-ci par le chef de l'Etat sont importants. Or, sa création s'inscrit dans la logique de limitation des pouvoirs de celui-ci. Le "devoir d'ingratitude "57, dont parle Robert Badinter, à l'égard de l'autorité de nomination, est encore loin d'être une réalité en Afrique et plus précisément au Togo. Même aux Etats-Unis d'Amérique, la Cour suprême est de tendance conservatrice ou progressiste selon que la majorité de ses membres est désignée par un Président conservateur ou un Président démocrate. Pour réduire donc les risques de récupération politique de la Cour constitutionnelle par le Président de la République, il faudra limiter l'importance du monde politique dans la désignation des membres de celle-ci. Dans ce sens, la formule suivante pourra être utilisée, si l'on limitait à sept (7) les membres de la Cour : deux personnalités ayant une grande expérience professionnelle, dont une proposée par le bureau de l'Assemblée nationale et une proposée par le Président de la République ; deux magistrats élus par leurs pairs ; un avocat élu par ses pairs ; un enseignant de la faculté de droit titulaire au moins d'un doctorat en droit public élu par ses pairs ; un représentant des Associations de Défense des Droits de l'Homme reconnu pour sa compétence en droit public. Le Président de la Cour sera désigné par ses pairs. On constate que cette formule confierait la désignation des 2/5 des membres de la Cour constitutionnelle togolaise à la société civile, ce qui est, à notre humble avis, un gage d'indépendance de la Cour. En tout état de cause, il faut préciser " qu'en réalité, il n'existe pas de procédure idéale [de désignation pouvant garantir automatiquement l'indépendance du juge constitutionnel]. Il y a toujours un risque de politisation ou de corporatisme. Seuls l'environnement démocratique et l'esprit d'indépendance des juges comptent "158.

54 Article roo de la constitution togolaise de 1992, version originale.

55 Article ror de la constitution togolaise de i992, version originale.

56 Article roo de la constitution togolaise de 1992 tel qu'issu de la révision de 2002 : « la Cour constitutionnelle est composée de neuf (og) membres désignés pour sept ans renouvelables. Trois sont désignés par le Président de la République dont un (01) en raison de ses compétences juridiques. Trois (03) sont élus par l'Assemblée nationale à la majorité des deux tiers (2/3) de ses membres. Ils doivent être choisis en dehors des députés. L'un d'entre eux doit être désigné en raison de ses compétences juridiques. Trois (03) sont élus par le sénat à la majorité des deux tiers (2/3) de ses membres. Ils doivent être choisis en dehors des sénateurs. L'un d'entre eux doit être désigné en raison de ses compétences juridiques".

57 Cité par Dominique Turpin, Droit constitutionnel, op. cit. p. 763.

58 Adama Kpodar, "Réflexion sur la justice constitutionnelle à travers le contrôle de constitutionnalité de la loi dans le nouveau 
Au plus, l'indépendance de la Cour constitutionnelle pourra être améliorée en renforçant le statut de ses membres. Actuellement, les membres de la Cour constitutionnelle sont nommés pour un mandat irrévocable de sept ans renouvelable indéfiniment. Si l'irrévocabilité prévue par le constituant togolais est satisfaisante, puisqu'elle protège le juge constitutionnel contre les humeurs de l'autorité de nomination, le renouvellement indéfini est de nature à compromettre l'indépendance de l'institution juridictionnelle. Normalement, l'irrévocabilité du mandat des membres de la Cour constitutionnelle togolaise devra être nécessairement couplée du non renouvellement de celui-ci, ce qui fera, comme le note le Professeur Charles EISENMANN, qu'ils n'auront " plus rien à craindre ni à attendre de l'autorité de nomination "59.

A côté de la juridiction constitutionnelle autonome, les Autorités administratives indépendantes $(\mathrm{AAI})^{60}$ ont également fait leur apparition à partir des années 1990 , afin de consolider les acquis démocratiques et de conduire la construction de l'Etat de droit $^{6 \mathrm{r}}$. Il s'agit, en effet, de la Commission Nationale de défense des Droits de l'Homme $(\mathrm{CNDH})^{62}$, de la Haute Autorité de l'Audiovisuel et de la Communication (HAAC), du Médiateur de la République (MR) ou de la Commission Electorale Nationale Indépendante (CENI). La CNDH a pour mission d'assurer la protection, la promotion et la défense des droits de l'homme, tandis que le MR est chargé de recevoir les réclamations concernant le fonctionnement des administrations de l'Etat des collectivités publiques territoriales, des établissements publics, dans leurs relations avec les administrés. Quant à la HAAC, elle est chargée de garantir et d'assurer la liberté et la protection de la presse et des autres moyens de communication de masse en veillant au respect de la déontologie en matière d'information, de communication et à l'accès équitable des partis politiques et des associations aux moyens officiels d'information et de communication. La CENI, de son côté, a été créée pour prendre en charge la gestion du processus électoral aux lieu et place du ministère de l'administration territoriale jugée très impartiale, afin de favoriser l'organisation d'élections libres et transparentes. Au regard de leurs attributions, on constate que certaines de ces institutions ( $\mathrm{CNDH}, \mathrm{MR})$ vont contribuer à limiter les violations des droits de l'homme, tandis que les autres (CENI, HAAC) vont réduire les risques de pérennisation du chef de l'Etat au pouvoir. Mais une fois encore, on constate que ces institutions n'ont pas été à la hauteur de tous les espoirs placés en eux. Il s'est révélé nécessaire de les affranchir davantage de la tutelle du chef de l'Etat. Ceci passera à coup sûr par une nouvelle lecture de leur statut, surtout des modalités de désignation de leurs membres qui, actuellement, sont tous ou presque désignés par le couple Parle-

constitutionnalisme : les cas du Bénin, du Mali, du Sénégal et du Togo ", op. cit., p. I42.

59 Cité par Jean Gicquel et Jean-Eric Gicquel, Droit Constitutionnel et institutions politiques, op. cit., p. 735.

6o Les AAI sont des institutions ayant pour mission "d'assurer l'impartialité de l'Etat face aux comportements de l'administration dans certains secteurs d'activité qui nécessitent un équilibre subtil entre l'intérêt général et les intérêts particuliers ou qui nécessitent à la fois grande technicité et impartialité "; cf. Bernard Chantebout, Droit constitutionnel, op. cit., p. 633.

6I Voir Abdoulaye Diarra, "Les autorités administratives indépendantes dans les Etats francophones d'Afrique noire : cas du Mali, du Sénégal et du Bénin ", op. cit.

62 Il faut tout de même mentionner que la CNDH présente la particularité d'être créée en I987, c'est-à-dire sous le régime du parti unique avant d'être constitutionnalisée par la Constitution de 1992. 
ment-Gouvernement. Pour renforcer leur indépendance il faudra limiter l'intervention du monde politique (Parlement, chef de l'Etat) dans la désignation des membres de ces organes en prévoyant un mode de désignation qui donnera une part plus importante à la société civile.

\section{Conclusion}

Le modèle constitutionnel envisagé vise à adapter le régime à la réalité politique en Afrique et plus particulièrement au Togo. En effet, on constate que malgré la structure bicéphale que le constituant prévoit, le Président de la République est à la fois chef de l'Etat et chef du Gouvernement. En réalité, depuis les années I990, le pouvoir exécutif n'a jamais fait l'objet de partage entre le chef de l'Etat et un Premier ministre. Dans ce sens, le bicéphalisme de l'exécutif est loin de faire recette, car le chef de l'Etat reste et demeure le principal pôle d'impulsion du pouvoir politique. Toutes les autres institutions lui restent soumises. D'ailleurs la constitution a été très vite révisée en 2002 dans une perspective de subordination du Premier ministre au chef de l'Etat. Non seulement son statut est rendu précaire, mais aussi les attributions du Premier ministre sont introuvables. Pis encore, le bicéphalisme a été à l'origine de multiples crises au sommet de l'Etat, liées tantôt à la désignation du Premier ministre, tantôt à la répartition du pouvoir exécutif entre ce dernier et le chef de l'Etat. Les faits restent têtus et nous obligent par conséquent à assumer la prééminence présidentielle. Ainsi, la structure monocéphale de l'exécutif d'inspiration présidentielle répond bien à cette réalité. Elle convient mieux, du moins à l'heure actuelle, à l'environnement politique africain. Seulement, il faut chercher à encadrer, ou à rationnaliser cette prééminence afin d'éviter une dérive autoritaire du pouvoir présidentiel. L'objectif n'est pas d'empêcher le Président de la République d'exercer ses pouvoirs, au contraire, il faut encadrer l'exercice du pouvoir présidentiel. Mais, contrairement au constituant de I990, il faut plutôt chercher la limitation du pouvoir présidentielle en dehors du pouvoir exécutif. Traditionnel contrepoids au pouvoir exécutif, il revient au Parlement de jouer ce rôle de frein à l'abus du pouvoir présidentiel. Seulement il faut renforcer son rôle et son statut en supprimant notamment le droit de dissolution confié au chef de l'Etat. Mais il convient aussi de mettre en contribution les autres organes de contrepouvoir, notamment les Cours constitutionnelles et les Autorités administratives indépendantes. Mais celles-ci ne pourront jouer efficacement ce rôle de contrepoids qu'une fois affranchies de la tutelle du pouvoir en place. C'est pourquoi une relecture de leur statut s'impose. Il s'avère également important de mettre un accent particulier sur la culture démocratique et l'éthique constitutionnelle, car comme le dit un grand observateur avisé du constitutionnalisme africain, "constitution, sans culture constitutionnelle, n'est que ruine de constitutionnalisme "63.

63 Jean du Bois de Gaudusson, "Constitution sans culture constitutionnelle n'est que ruine du constitutionnalisme », Démocratie et liberté : tension, dialogue, confrontation, Mélanges en l'honneur de Slobodan Milacic, Bruylant, 2008, p. 348. 